\title{
Adenosarcoma mulleriano del cérvix: reporte de caso con manejo conservador
}

\author{
Mullerian adenosarcoma of the cervix: case report with conservative management
}

Javier A. Teco-Cortes ${ }^{1}$, Peter Grube-Pagola ${ }^{1 *}$, Vicente A. Saldaña-Quiroz ${ }^{2}$ y Georgia Alderete-Vázquez ${ }^{3}$

${ }^{1}$ Departamento de Anatomía Patológica, Instituto de Investigaciones Médico-Biológicas, Universidad Veracruzana; ${ }^{2}$ Departamento de Ginecología y Obstetricia, Hospital Star Médica Veracruz; ${ }^{3}$ Consultorio de Patología Dra. Isabel Ruiz Juárez, Veracruz. Ver. México

\section{Resumen}

Antecedentes: El adenosarcoma mulleriano (AM) es una neoplasia ginecológica rara, de bajo potencial maligno, con crecimiento bifásico, constituida por un elemento epitelial benigno y otro mesenquimatoso maligno. Se presenta en todas las edades, pero predomina en mujeres posmenopáusicas. La localización cervical de los AM es poco frecuente; sin embargo, se asocia a una presentación en mujeres jóvenes. El diagnóstico se realiza mediante estudio anatomopatológico de la lesión e inmunohistoquímica. El pronóstico es generalmente bueno, aunque la tasa de recidiva es alta. Caso clínico: Presentamos el caso de una paciente de 27 años que acudió a consulta ginecológica con sangrado y flujo transvaginal. En la exploración ginecológica se identificó una lesión polipoide originada en el cérvix, la cual se extirpó por torsión y fue diagnosticada como AM cervical. Posteriormente se realizó conización cervical debido a que la paciente rechazó la histerectomía. Conclusión: El AM cervical es una neoplasia poco frecuente que tiene una tasa de recidiva que puede llegar hasta al $50 \%$ de los casos, por lo que es necesario un seguimiento estrecho. La escisión local puede ser considerada en pacientes sin factores de mal pronóstico y que deseen conservar su fertilidad.

PALABRAS CLAVE: Sarcoma uterino. Adenosarcoma mulleriano cervical. Cérvix. Neoplasia ginecológica.

\begin{abstract}
Background: Mullerian adenosarcoma is a rare gynecological malignancy with a low malignant potential, with biphasic growth, consisting of a benign epithelial element and a malignant mesenchymal element. It occurs in all ages predominating in postmenopausal women. Cervical localization of Mullerian adenosarcomas is rare; however, it is associated with a presentation in young women. The diagnosis is made by anatomopathological study of the lesion and immunohistochemistry. The prognosis is generally good although the recurrence rate is high. Clinical case: We present the case of a 27-year-old patient who attended a gynecological consultation with bleeding and transvaginal flow. During the gynecological examination, a polypoid lesion originating in the cervix was identified, which was removed by torsion and was diagnosed as Mullerian cervical adenosarcoma. Subsequently, a cervical cone was performed because the patient refused hysterectomy. Conclusions: Mullerian cervical adenosarcoma is a rare neoplasm with a recurrence rate that can reach up to $50 \%$ of cases, so close follow-up is necessary. A local excision can be considered in patients without poor prognosis factors and who wish to preserve their fertility.
\end{abstract}

KEY WORDS: Uterine sarcoma. Cervical Mullerian adenosarcoma. Cervix. Gynecological neoplasia.

\author{
Correspondencia: \\ *Peter Grube-Pagola \\ Instituto de Investigaciones Médico-Biológicas \\ Iturbide $\mathrm{s} / \mathrm{n}$, Zona Centro \\ C.P. 91700 , Veracruz, Ver., México \\ E-mail: grubejr78@gmail.com
}

Fecha de recepción: 04-01-2018

Fecha de aceptación: 20-04-2018

DOI://dx.doi.org/10.24875/CIRU.M18000027
Cir Cir. 2018;86:191-195

Contents available at PubMed www.cirugiaycirujanos.com 


\section{Antecedentes}

El adenosarcoma mulleriano (AM) fue descrito por Clement y Scully en 1974 en el cuerpo uterino, el cual ha sido el sitio de presentación más frecuente, aunque se puede presentar en otras localizaciones como el cérvix, el ovario, la vagina, la trompa uterina y la serosa intestinal. Representa el $5-7 \%$ de los sarcomas uterinos ${ }^{1-3}$, y el $A M$ del cérvix representa el $2 \%$ de todos los $\mathrm{AM}^{4}$. El AM cervical se presenta con mayor frecuencia en mujeres jóvenes, reportándose hasta un tercio de estos en pacientes entre los $10 \mathrm{y}$ los 15 años de edad, teniendo una marcada diferencia con su homólogo en el cuerpo uterino, el cual suele presentarse en mujeres posmenopaúsicas ${ }^{5}$. Son tumores de etiología indeterminada, con potencial maligno bajo, caracterizados por un componente epitelial benigno y otro mesenquimatoso maligno, considerado por algunos autores un punto intermedio en el camino entre el adenofibroma y el carcinosarcoma. Clínicamente suelen presentarse con sangrado o descarga transvaginal, y en ocasiones puede identificarse una masa que protruye hasta la vagina ${ }^{4,5}$. El diagnóstico definitivo se realiza por medio del estudio histopatológico e inmunohistoquímico. El tratamiento utilizado más frecuentemente es la histerectomía con salpingooforectomía bilateral; sin embargo, el manejo de cada caso debe ser individualizado. El pronóstico en general es bueno, aunque su comportamiento biológico en ocasiones puede ser agresivo, siendo la invasión profunda al miometrio y el sobrecrecimiento sarcomatoso los principales factores de mal pronóstico. Las metástasis se presentan en el $2 \%$ de los casos, pero la tasa de recidiva local es mucho más alta, siendo hasta del $50 \%{ }^{2,3}$.

El objetivo de la presentación de este caso es destacar la importancia de un diagnóstico y un seguimiento adecuados, debido a la alta tasa de recidiva, así como la individualización del tratamiento, de acuerdo con todos los principios de la ética médica.

\section{Caso clínico}

Mujer de 27 años que acudió a consulta ginecológica por presentar sangrado y flujo transvaginal. Sin antecedentes heredofamiliares ni personales de importancia; en los antecedentes gineco-obstétricos refirió menarca a los 13 años, con ciclos regulares, sangrado moderado y duración de 3 a 5 días sin asociarse a ningún síntoma. Nulípara, con vida sexual activa y sin uso de método anticonceptivo.

Inició su padecimiento actual 3 meses antes de acudir a consulta, con flujo transvaginal de mal olor y de aspecto blanquecino a amarillento, acompañado de sangrado transvaginal intermitente, leve a moderado, no relacionado con la menstruación ni con ningún factor traumático; no presentó otra sintomatología.

Se realizó colposcopia, la cual mostró una lesión que protruía a través del agujero cervical externo con zonas de aspecto ulcerado (Fig. 1). Se realizó toma de biopsia y citología exfoliativa; el reporte citológico fue de inflamación crónica y aguda con fibrina, mientras que el reporte de la biopsia fue de neoplasia bifásica epitelio-mesenquimatosa ulcerada con atipia del componente estromal. Se le realizaron exámenes de laboratorio, que reportaron parámetros dentro de la normalidad (hemoglobina $11.55 \mathrm{~g} / \mathrm{dl}$, hematocrito $34.74 \%$, plaquetas $260,000 / \mu \mathrm{l}$, leucocitos $9090 / \mu \mathrm{l}$, tiempo de protrombina 11.5 segundos, tiempo parcial de tromboplastina 29.7 segundos, glucosa $70 \mathrm{mg} / \mathrm{dl}$, resto de química sanguínea y perfil de lípidos sin alteraciones).

Posteriormente se realizó ultrasonido abdominal y transvaginal, el cual mostró un útero de $73.7 \times 34.5$ $\times 51.2 \mathrm{~mm}$, miometrio sin alteraciones aparentes, endometrio de $8.8 \mathrm{~mm}$ con engrosamiento en el tercio medio compatible con mioma submucoso, cérvix con morfología deformada a expensas de proceso ocupativo sólido con leve vascularidad (Doppler color), y ovarios con múltiples quistes.

Se efectuó exploración bajo anestesia, encontrando una lesión exofítica polipoide de $3 \mathrm{~cm}$, con pedículo proveniente del canal endocervical; la lesión se extirpó completamente por torsión. Se realizó histerometría directa de $7 \mathrm{~cm}$ con legrado-biopsia de endometrio, y finalmente exploración bimanual con útero y anexos sin masas palpables, y tabiques y parametrios libres.

La lesión polipoide fue de color café grisáceo, consistencia ahulada, superficie nodular, midiendo $3.5 \times$ $2.5 \times 2.1 \mathrm{~cm}$. Al corte fue homogénea, de color amarillo grisáceo y de aspecto mixoide (Fig. 2). El estudio histopatológico mostró una lesión bifásica con patrón de crecimiento sólido constituido por haces celulares irregulares con zonas hipercelulares e hipocelulares interrumpidas por conductos de diversos tamaños, de pequeños a grandes, los cuales daban un patrón de aspecto foliáceo (Fig. 3). Se realizaron reacciones de inmunohistoquímica para receptores de estrógeno, receptores de progesterona, actina de músculo liso y WT1, las cuales fueron positivas en el componente estromal, mostrando además CD10 


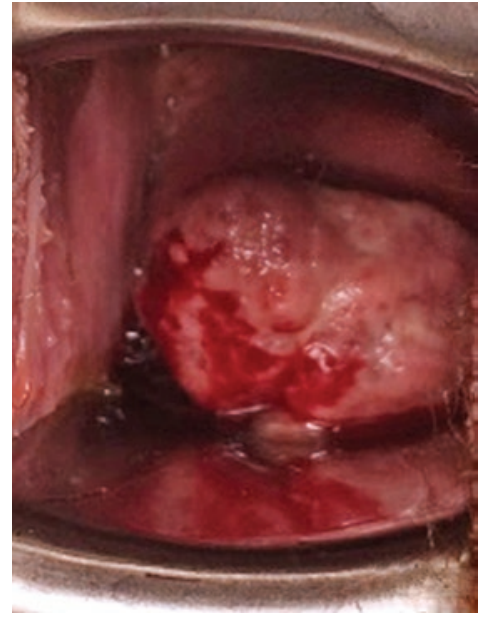

Figura 1. Aspecto macroscópico de la neoplasia con superficie ulcerada y áreas hemorrágicas.
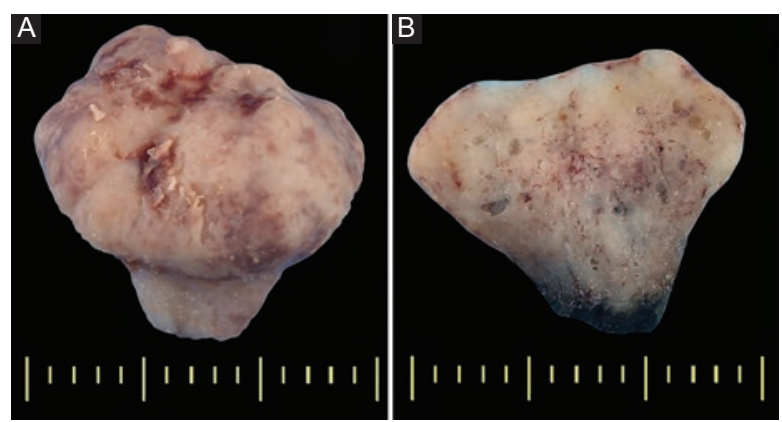

Figura 2. A: aspecto macroscópico de la neoplasia: polipoide con base pediculada, y la superficie muestra tejido de granulación. B: al corte es sólida, con pequeños quistes, y con vascularidad acentuada.

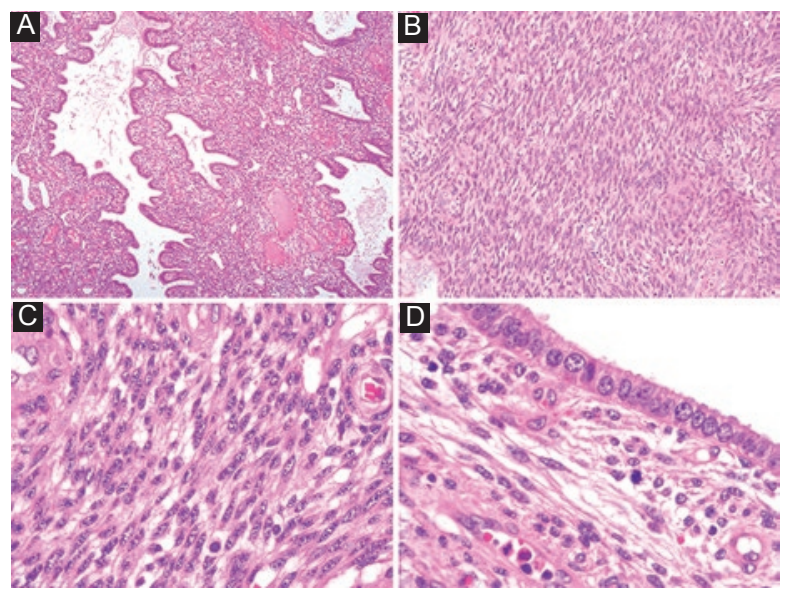

Figura 3. A: aspecto microscópico con patrón foliáceo (hematoxilina y eosina, 2x). B: en las áreas sólidas la neoplasia crece formando haces celulares cortos (hematoxilina y eosina, 10x). C: las células estromales son ahusadas, con citoplasma de bordes poco definidos y eosinófilos; los núcleos son alargados, con cromatina granular dispersa (hematoxilina y eosina, 40x). D: detalle del componente epitelial, el cual es cúbico simple sin atipias; en el estroma subyacente se aprecia mitosis atípica (hematoxilina y eosina, 40x). positivo en las células neoplásicas adyacentes al componente epitelial; el índice de proliferación con Ki67 fue de 5-8\% (Fig. 4). El diagnóstico fue de adenosarcoma mulleriano. La biopsia de endometrio mostró cambios de hiperplasia simple sin atipias.

Se propuso a la paciente tratamiento con histerectomía más salpingooforectomía bilateral o tratamiento conservador con conización y seguimiento estrecho debido al riesgo de recidiva, optando por este último debido al deseo de gestación. Se le realizó conización cervical sin complicaciones; el estudio patológico reportó cervicitis crónica acentuada y aguda focal con metaplasia escamosa madura endocervical, sin presencia de neoplasia. Se egresó a la paciente y se estructuró un plan de seguimiento estrecho de forma ambulatoria; en la actualidad, a 6 meses y con estudio de tomografía, la paciente se encuentra libre de actividad tumoral.

\section{Discusión}

Los sarcomas uterinos son un grupo de neoplasias de origen mesenquimatoso, representando el $8 \%$ de las neoplasias malignas del útero ${ }^{6}$. Entre ellos se encuentra el $\mathrm{AM}$, un tumor con potencial maligno bajo que representa el $5-8 \%$ de los sarcomas uterinos ${ }^{7}$, descrito originalmente por Clement y Scully en $1974^{1,2}$. Se origina con mayor frecuencia en el endometrio del cuerpo uterino, pero también puede desarrollarse en el ovario, el cérvix ${ }^{1,5}$, el retroperitoneo y el intestino ${ }^{8}$. El AM del cérvix representa el 2-9\% de todos los AM7.

El AM del cérvix es una neoplasia bifásica, ya que se compone de epitelio benigno y estroma maligno 4 . Su etiología es desconocida, aunque algunos casos se han asociado a uso de anticonceptivos orales, tamoxifeno ${ }^{7}$, hiperestrogenismo endógeno ${ }^{2}$, antecedentes de radiación pélvica y antecedentes neoplási$\cos ^{1}$; en nuestro caso, la paciente no refirió ningún antecedente de importancia, aunque se detectó ovario poliquístico, un hallazgo asociado a alteraciones endocrinológicas.

El AM cervical puede presentarse en mujeres de cualquier edad, pero es más común en las jóvenes, con una media de 27 años, como en nuestro caso, siendo reportados el $30 \%$ en pacientes entre los 10 y los 15 años?.

La presentación clínica característica es con sangrado transvaginal ${ }^{1,4,9}$; en nuestra paciente, además, se asoció con vaginosis bacteriana, por lo que el tratamiento inicial fue a base de antibioticoterapia. Otras 


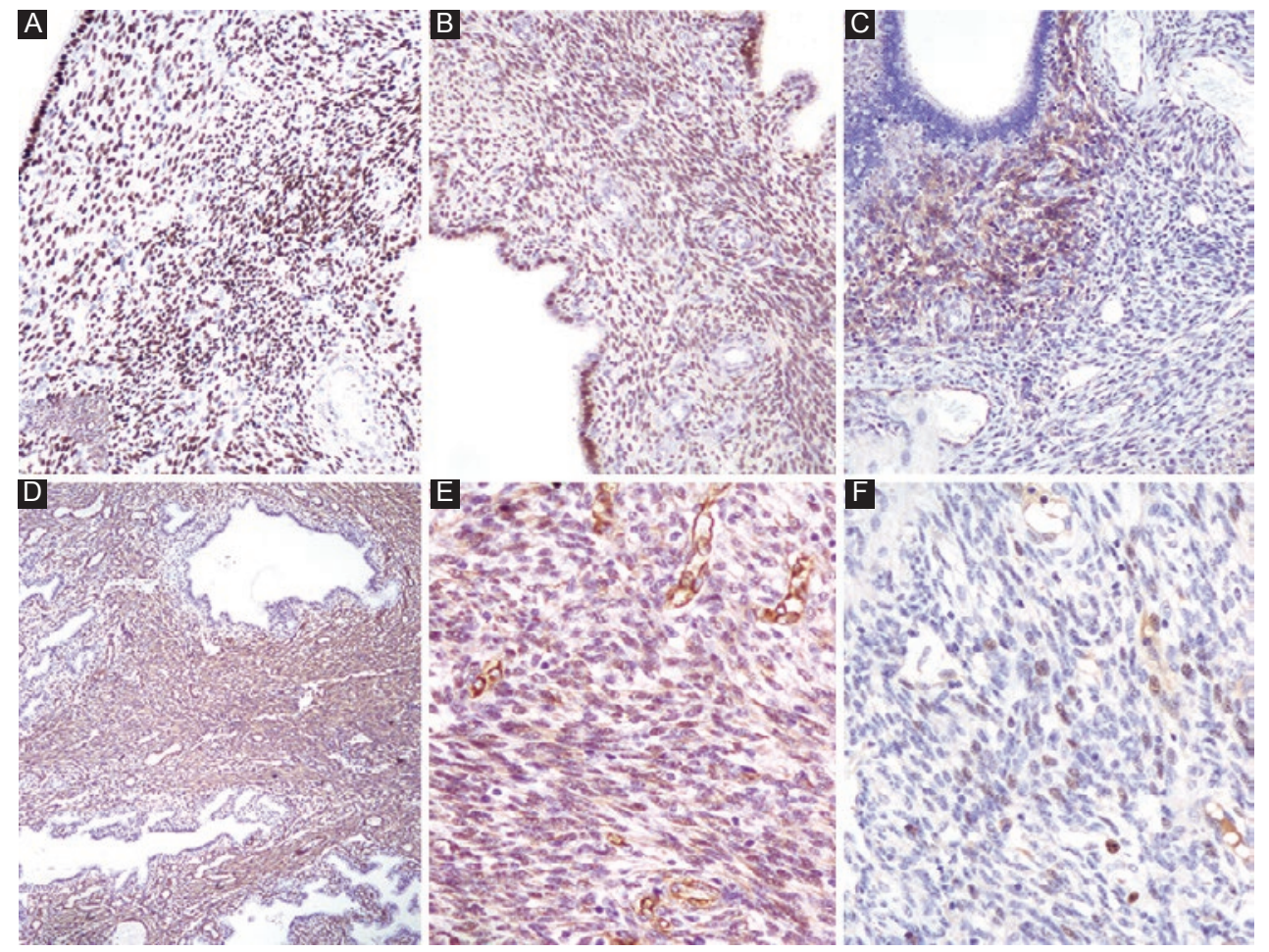

Figura 4. Inmunorreacciones positivas. A: receptores de estrógeno. B: receptores de progesterona. C: CD10 en capa de cambio. D: antiactina de músculo liso. E: WT1. F: Ki67 en el 5-8\% en células neoplásicas.

alteraciones menos comunes asociadas a estos casos son dolor abdominal, infecciones del tracto urinario, prolapso uterino e incluso ascitis' ${ }^{1}$. La exploración física suele revelar una masa polipoide que protruye a través de un conducto cervical dilatado, en ocasiones llegando hasta la vagina ${ }^{1,2,5}$, la cual fue identificada en nuestro caso al realizar una exploración ginecológica bajo anestesia, que permitió la escisión del tumor y la toma de biopsia endometrial.

Los exámenes de laboratorio fueron normales en nuestra paciente; no hay datos reportados de alteraciones asociadas a estos casos. Los estudios de imagen revelan masas polipoides bien delimitadas que pueden protruir por el canal endocervical o hacia la cavidad endometrial. Se pueden observar zonas de apariencia quística y septos junto a un componente sólido heterogéne $0^{6,10}$, con un realce similar al del endometrio tras la administración de medio de contraste ${ }^{2}$, siendo utilizado en este caso ultrasonido (y Doppler color), el cual evidenció la lesión con alguna vascularidad.

Macroscópicamente, el tamaño de la lesión es variable (promedio de $5 \mathrm{~cm}$ ), y es típica una masa solitaria, sésil o pedunculada, polipoide o papilar, que se proyecta hacia su cavidad (endocervical o miometrial), presentando una consistencia desde suave hasta firme (de gelatinosa a carnosa o gomosa) ${ }^{1}$. Al corte se pueden observar quistes de tamaño variable, zonas de hemorragia focal y necrosis, con un margen tumoral bien definido; en ocasiones, se forman múltiples pólipos ${ }^{1,3}$. En nuestro caso se presentó como una lesión de $3.5 \mathrm{~cm}$, predominantemente sólida, de aspecto mixoide y ocasionales quistes.

Microscópicamente se observa una neoplasia bifásica con un componente epitelial benigno y otro mesenquimatoso maligno; a bajo aumento, este patrón foliáceo es similar al tumor phylodes de mama². El epitelio glandular en general es de tipo endometrioide con células de abundante citoplasma eosinófilo, en ocasiones acompañado de diferenciación mucosa y escamosa ${ }^{2,3}$; puede presentar atipia nuclear (núcleos grandes, pleomorfismo, engrosamiento de cromatina, hipercromasia, nucléolos grandes) y en ocasiones forma líneas entre el estroma, dándole una arquitectura papilar o pseudovellosa'. El estroma se compone de células redondeadas o fusiformes, con diversos grados de atipia y núcleos hipercromáticos, las cuales suelen concentrarse alrededor de los elementos glandulares formando manguitos, también denominados capa de cambio,3; puede presentar proyecciones en la luz del componente glandular ${ }^{1}$. Según los criterios de la Organización Mundial de la Salud, el componente estromal debe presentar un índice mitótico $\geq 2$ mitosis en 10 campos a gran aumento para diferenciarlo del adenofibroma. La mayoría 
de los adenosarcomas contienen un componente mesenquimatoso homogéneo, pero hasta el $25 \%$ pueden presentar elementos heterólogos (rabdomiosarcoma, condrosarcoma, liposarcoma, etc.) o un patrón de sobrecrecimiento sarcomatoso $0^{2,5,11}$, los cuales no se presentaron en nuestro caso.

La inmunohistoquímica revela la expresión de marcadores de diferenciación celular (estrógenos y progesterona, CD10 y WT1) en el componente estromal. El CD10 particularmente se expresa en la capa de cambio. Los marcadores de proliferación celular (Ki67) suelen mostrar bajos índices. Sin embargo, en zonas sarcomatosas la proliferación celular es mayor, así como la pérdida en la expresión de los marcadores de diferenciación celular, siendo el inmunoperfil similar al del sarcoma uterino indiferenciado. En algunas ocasiones se presenta DNA aneuploide ${ }^{2,3,12}$. Nuestro caso fue confirmado por inmunohistoquímica, presentando un patrón bien diferenciado.

Entre los diagnósticos diferenciales histológicos se incluyen tumores benignos, como el adenofibroma, el pólipo cervical atípico o el adenomioma del cérvix, y neoplasias malignas como el adenosarcoma uterino con extensión al cérvix, el carcinosarcoma y el rabdomiosarcoma embrionario $0^{5,9}$.

El diagnóstico diferencial principal es el adenofibroma, pero existe controversia en este aspecto debido a que la morfología, el inmunoperfil y el pronóstico son similares, presentando diferencia solo cuando el adenosarcoma tiene sobrecrecimiento estromal. Se han reportado también casos de adenofibroma con comportamiento biológico maligno, motivo por el cual se propone que este tumor es un adenosarcoma bien diferenciado más que un tumor diferente del adenosarcoma ${ }^{3,13}$.

No existe evidencia del tratamiento más eficaz, y siguiendo lo estudiado en el AM del cuerpo uterino, el tratamiento más utilizado es la histerectomía con salpingooforectomía; sin embargo, como tratamiento alternativo se ha utilizado la cirugía con preservación de la fertilidad con confirmación de márgenes tumorales negativos ${ }^{1,4,5,7,11}$. Las terapias adyuvantes (quimioterapia o radioterapia) no han demostrado beneficio en estos casos $^{14}$. Debido a que nuestra paciente rechazó la histerectomía por el deseo de gestación, se optó por la escisión local del tumor y la conización cervical para confirmar los márgenes negativos. En cualquier caso, la tasa de recidiva es alta, llegando al $25 \%$ en las pacientes tratadas con histerectomía y hasta el $50 \%$ en las tratadas con escisión local ${ }^{1{ }^{17}}$; la recurrencia es frecuente a largo plazo, por lo que debe realizarse un seguimiento estrecho $0^{1,5}$.

El sobrecrecimiento sarcomatoso y la invasión miometrial son los principales factores de mal pronóstico ${ }^{1,7,14}$, además de la presencia de elementos heterólogos, alto índice mitótico, necrosis y extensión extrauterina ${ }^{5,10}$. La afectación de ganglios linfáticos es del $3-4 \%$ y la supervivencia general a 5 años es del $79 \%{ }^{14}$. En nuestro caso no se encontraron factores de mal pronóstico, pero aun así se seguirá de forma estrecha a la paciente debido a la probabilidad de recidiva.

En conclusión, el AM cervical es una neoplasia de bajo potencial maligno y alta tasa de recidiva, presentando un comportamiento biológico incierto, por lo que es necesario un seguimiento a largo plazo incluso después de la histerectomía. Aun así, es factible considerar un tratamiento conservador en aquellas pacientes que deseen preservar la fertilidad y sin factores de mal pronóstico, estableciendo un seguimiento estrecho.

\section{Bibliografía}

1. Clement PB, Scully RE. Mullerian adenosarcoma of the uterus: a clinicopathologic analysis of 100 cases with a review of the literature. Hum Pathol. 1990;21:363-81.

2. Friedlander ML, Covens A, Glasspool RM, Hilpert F, Kristensen G, Kwon S, et al. Gynecologic Cancer InterGroup (GCIG) consensus review for Mullerian adenosarcoma of the female genital tract. Int J Gynecol Cancer. 2014;24:S78-82.

3. McCluggage WG. Mullerian adenosarcoma of the female genital tract. Adv Anat Pathol. 2010;17:122-9.

4. Shinnick JK, Kumar N, Beffa L, Miller K, Friedman MA, Kalife E, et al. Management of low-grade cervical Müllerian adenosarcoma in a 14-yearold girl. J Pediatr Adolesc Gynecol. 2017;30:652-4.

5. Seagle BL, Falter KJ $2^{\text {nd }}$, Lee SJ, Frimer M, Samuelson R, Shahabi S. Mullerian adenosarcoma of the cervix: report of two large tumors with sarcomatous overgrowth or heterologous elements. Gynecol Oncol Case Rep. 2014;9:7-10.

6. Santos P, Cunha TM. Uterine sarcomas: clinical presentation and MRI features. Diagn Interv Radiol. 2015;21:4-9.

7. Kanayama S, Nakamura M, Oi H, Sugimoto S, Sasaki Y, Uchiyama T, et al. Case report of successful childbearing after conservative surgery for cervical Mullerian adenosarcoma. Case Rep Obstet Gynecol. 2017; 2017:4187416.

8. Daskalaki A, Xenaki S, Athanasakis E, Chrysos E, Chalkiadakis G. Advanced mesodermal (Müllerian) adenosarcoma of the ovary: metastases to the lungs, mouth, and brain. Case Rep Surg. 2015;2015:403431.

9. Podduturi V, Pinto KR. Mullerian adenosarcoma of the cervix with heterologous elements and sarcomatous overgrowth. Proc (Bayl Univ Med Cent). 2016;29:65-7.

10. Morales DA, Medina ML, Trujillo LM, Beltrán MI, Dulcey IC. Müllerian adenosarcoma of the uterine cervix with sarcomatous overgrowth: a case report of aggressive disease in a young patient. Int $\mathrm{J}$ Surg Case Rep. 2016;27:155-61.

11. Ramos P, Ruiz A, Carabias E, Piñero I, Garzón A, Álvarez I. Müllerian adenosarcoma of the cervix with heterologous elements: report of a case and review of the literature. Gynecol Oncol. 2002;84:161-6.

12. Soslow RA, Ali A, Oliva E. Mullerian adenosarcomas: an immunophenotypic analysis of 35 cases. Am J Surg Pathol. 2008;32:1013-21.

13. Gallardo A, Prat J. Mullerian adenosarcoma, a clinicopathologic and immunohistochemical study of 55 cases challenging the existence of adenofibroma. Am J Surg Pathol. 2009;33:278-88.

14. Seagle $B L$, Kanis M, Strohl AE, Shahabi S. Survival of women with Mullerian adenosarcoma: a National Cancer Data Base study. Gynecol Oncol. 2016;143:636-41. 